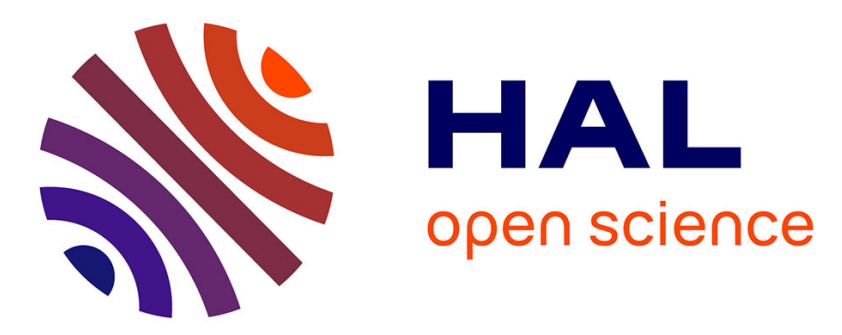

\title{
Elasticity: Thermodynamic Treatment
}

André Zaoui, Claude Stolz

\section{To cite this version:}

André Zaoui, Claude Stolz. Elasticity: Thermodynamic Treatment. Encyclopedia of Materials: Science and Technology, Elsevier Science, pp.2445-2449, 2000, 10.1016/B0-08-043152-6/00436-8 . hal00112293

\section{HAL Id: hal-00112293 \\ https://hal.science/hal-00112293}

Submitted on 31 Oct 2018

HAL is a multi-disciplinary open access archive for the deposit and dissemination of scientific research documents, whether they are published or not. The documents may come from teaching and research institutions in France or abroad, or from public or private research centers.
L'archive ouverte pluridisciplinaire HAL, est destinée au dépôt et à la diffusion de documents scientifiques de niveau recherche, publiés ou non, émanant des établissements d'enseignement et de recherche français ou étrangers, des laboratoires publics ou privés. 


\section{Elasticity: Thermodynamic Treatment}

The elastic behavior of materials is usually described by direct stress-strain relationships which use the fact that there is a one-to-one connection between the strain and stress tensors $\varepsilon$ and $\sigma$; some mechanical elastic potentials can then be defined so as to express the stress tensor as the derivative of the strain potential with respect to the strain tensor, or conversely the latter as the derivative of the complementary (stress) potential with respect to the former.

Nevertheless, these potentials are restricted to specific thermodynamic conditions. This purely mechanical treatment may be generalized and improved by integrating its temperature dependence and by connecting the elastic potentials with the classical thermodynamic functions within a consistent thermodynamic framework. This leads to a more general definition of thermoelasticity (Sect. 1), which can be used for a better understanding of the properties of the elastic moduli (Sect. 2), for a natural definition of rubber elasticity (Sect. 3), and for the prediction of the effective thermal expansion coefficients of heterogeneous materials (Sect. 4).

\section{Thermodynamic Definition of Thermoelasticity}

\subsection{General Case}

The thermodynamic framework relies on the two fundamental (conservation) classical principles, for which local expressions can be derived from the global ones (Germain et al. 1983). Infinitesimal strains as well as quasi-static evolutions only are considered. For any given body, $V$, subjected to purely thermodynamic processes, the first principle expresses the fact that, whatever the mechanical behavior, the variation of the total (i.e., internal plus kinetic) energy is equilibrated by the variation of the mechanical work of the applied forces and by the variation of the heat received by the body.

When thermal conduction only occurs through the boundary $\partial V$, with $\vec{q}$ the heat flux vector and $\vec{n}$ the outward unit normal, and with $r$ denoting the specific heat production rate, the global heat rate, $Q$, is given by $Q=\int_{V} r \mathrm{~d} V-\int \partial_{V} \vec{q} \cdot \vec{n} \mathrm{~d} S=\int_{V}(r-\operatorname{div}(\vec{q})) \mathrm{d} V$. Taking into account the equilibrium equations, which are used to connect the stress field with the exterior loading, the local expression of the first principle finally reads

$$
\rho \dot{e}=\sigma: \dot{\varepsilon}+r-\operatorname{div}(\vec{q})
$$

where $\rho$ is the mass per unit volume and $e$ the internal energy per unit mass. Here, variables with a dot above them denote time derivatives and, for second-order tensors $\mathbf{a}$ and $\mathbf{b}$, the notation $\mathbf{a}: \mathbf{b}$ represents $\mathbf{a}: \mathbf{b}=$ $\sum_{i j} a_{i j} b_{i j}=a_{i j} b_{i j}$.
A similar treatment can be applied to the second principle that relates to the existence of an absolute temperature, $T$, and of a thermodynamic function, the entropy, obeying a constitutive inequality ensuring the positivity of the dissipated energy. This leads to the following local inequality:

$$
\rho \dot{s}+\operatorname{div}\left(\frac{\vec{q}}{T}\right)-\frac{r}{T} \geqslant 0
$$

where $s$ is the entropy per unit mass. Elimination of the thermal source, $r$, between Eqns. (1) and (2) and use of the free energy per unit mass, $f=e-T s$, lead to the so-called Clausius-Duhem inequality

$$
\Phi=\sigma: \dot{\varepsilon}-\rho(\dot{f}+s \dot{T})-\frac{\vec{q}}{T} \cdot \vec{\nabla} T \geqslant 0
$$

which expresses the non-negativity of the dissipation, $\Phi$, per unit volume. Note that the total dissipation, $\Phi$, includes both an 'intrinsic' part, which is directly connected to the mechanical behavior, and a thermal part $(-(\vec{q} / \mathrm{T}) \cdot \vec{\nabla} T)$, associated with conduction.

\subsection{Thermoelasticity}

Thermoelastic behavior is defined by stating that the thermodynamic functions $e$ and $s$ as well as the stress tensor, $\sigma$, are one-to-one functions of the independent state variables $\varepsilon$ and $T$. In this case, the intrinsic dissipation does not depend on $\vec{\nabla} T$ in Eqn. (3). This means that both the intrinsic and the thermal dissipation must be non-negative separately. From the definition

$$
\dot{f}=\frac{\partial f}{\partial \varepsilon_{i j}} \dot{\varepsilon}_{i j}+\frac{\partial f}{\partial T} \dot{T}
$$

it follows for any evolution $(\dot{\varepsilon}, \dot{T})$ from the equilibrium state $(\varepsilon, T)$ :

$$
\left(\sigma_{i j}-\rho \frac{\partial f}{\partial \varepsilon_{i j}}\right) \dot{\varepsilon}_{i j}-\rho\left(s+\frac{\partial f}{\partial T}\right) \dot{T} \geqslant 0
$$

The independent variables $\dot{\varepsilon}$ and $\dot{T}$ may have arbitrary values, including zero; this results in the following relationships:

$$
\sigma=\rho \frac{\partial f}{\partial \varepsilon}(\varepsilon, T), \quad \text { i.e., } \sigma_{i j}=\rho \frac{\partial f}{\partial \varepsilon_{i j}} ; \quad s=-\frac{\partial f}{\partial T}(\varepsilon, T)
$$

which are the constitutive equations of thermoelasticity (Salençon 1995).

The free energy, $f(\varepsilon, T)$, from which the mechanical behavior can be derived completely for any thermodynamic conditions, can be considered as a thermo- 
dynamic potential, which is more general and powerful than the mechanical strain or stress potentials. The second expression of Eqn. (5) can be used, in association with the heat equation and boundary conditions, to derive the coupled thermomechanical evolution of a thermoelastic body.

An alternative treatment, relying on the state description through the variables $\sigma$ (instead of $\varepsilon$ ) and $T$, can be developed by using the stability of any deformed equilibrium state. It makes use of the potential $f^{*}$ defined by $f^{*}=(1 / \rho) \sigma: \varepsilon-f$. The associated constitutive equations are then

$$
\varepsilon_{i j}=\rho \frac{\partial f^{*}}{\partial \sigma_{i j}}(\sigma, T), \quad s=\frac{\partial f^{*}}{\partial T}(\sigma, T)
$$

\section{Elastic Moduli and Compliances}

\subsection{Symmetry}

When the potentials $f(\varepsilon, T)$ or $f^{*}(\sigma, T)$ are quadratic, the usual case of linear thermoelasticity is recovered: the constitutive equations (Eqns. (5) and (6)) yield a linear relationship between $\varepsilon$ and $\sigma$ through the classical elastic moduli $\mathbf{C}$ and compliances $\mathbf{S}$, respectively. When this is not the case, the same equations can be used to connect linearly infinitesimal variations of stress and strain through "tangent" moduli $\mathbf{L}$ and compliances $\mathbf{M}$. Their thermodynamic definition ensures the symmetry of these fourth-order tensors.

For example, for an isothermal evolution, $\mathbf{L}$ and $\mathbf{M}$ are simply given by the partial derivatives, at constant temperature, $\partial \sigma / \partial \varepsilon$ and $\partial \varepsilon / \partial \sigma$. Specification of the indices and use of Eqns (5) and (6) lead to

$L_{i j k l}=\frac{\partial \sigma_{i j}}{\partial \varepsilon_{k l}}=\rho \frac{\partial^{2} f}{\partial \varepsilon_{i j} \partial \varepsilon_{k l}}, \quad M_{i j k l}=\frac{\partial \varepsilon_{i j}}{\partial \sigma_{k l}}=\rho \frac{\partial^{2} f^{*}}{\partial \sigma_{i j} \partial \sigma_{k l}}$

Classical properties of second-order derivatives ensure that the isothermal elastic tangent moduli and compliances exhibit the diagonal symmetry

$$
L_{i j k l}=L_{k l i j}, \quad M_{i j k l}=M_{k l i j}
$$

This result is, of course, also valid for $\mathbf{C}$ and $\mathbf{S}$ in the case of linear elasticity.

\subsection{Isothermal and Adiabatic Compliances}

The elastic moduli and compliances depend on the thermodynamic regime. This can be illustrated by comparing isothermal ( $T$ constant) $\mathbf{M}^{\text {iso }}$ and adiabatic ( $s$ constant) $\mathbf{M}^{\text {ad }}$ compliances (François et al. 1998). They are defined by

$\mathrm{d} \varepsilon=\mathbf{M}^{\mathrm{iso}}: \mathrm{d} \sigma, \quad \mathrm{d} T=0 ; \quad \mathrm{d} \varepsilon=\mathbf{M}^{\mathrm{ad}}: \mathrm{d} \sigma, \quad \mathrm{d} s=0$
The isothermal compliances are given by Eqn. (7); the adiabatic ones result from Eqn. (6) and from the condition $\mathrm{d} s=0$. When the temperature dependence of $\rho$ is neglected, the order of magnitude of the difference between $\mathbf{M}^{\text {iso }}$ and $\mathbf{M}^{\text {ad }}$ can be estimated by assimilating $\varepsilon$ to the thermal strain, $\varepsilon^{\text {th }}=\alpha \Delta T$, so that $\partial \varepsilon / \partial T \approx \alpha$, and $\partial s / \partial T$ to $C_{\mathrm{p}} / T$, with $\alpha$ being the thermal expansion tensor and $C_{\mathrm{p}}$ the specific heat at constant stress.

This results in

$$
M_{i j k l}^{\mathrm{iso}}-M_{i j k l}^{\mathrm{ad}} \approx \frac{T}{\rho C_{\mathrm{p}}} \alpha_{i j} \alpha_{k l}
$$

(note that $\mathbf{M}^{\text {ad }}$ also exhibits diagonal symmetry). For isotropic materials $\left(\alpha_{i j}=\alpha \delta_{i j}\right.$, where $\delta_{i j}$ is the Kronecker symbol), and usual conditions, this proves that the relative difference between isotropic and adiabatic compliances hardly exceeds a few percent. However, this difference, which can only be understood from the thermodynamic treatment of elasticity, could have significant consequences in some extreme cases (dynamics, very high pressure or temperature variations, etc.).

\section{Crystal vs. Rubber Elasticity}

From Eqn. (5) and the relationship between $f, e$, and $s$, the constitutive equations of thermoelasticity can be put in the form

$$
\sigma=\rho \frac{\partial f}{\partial \varepsilon}(\varepsilon, T)=\rho \frac{\partial e}{\partial \varepsilon}-\rho T \frac{\partial s}{\partial \varepsilon}
$$

This expression clearly shows that thermoelasticity results from two sources: the strain dependence of the internal energy and that of the entropy. According to the materials under consideration, the first source or the second can be predominant:

(i) some materials, especially crystals and polycrystals, cannot deform very much in the elastic range and there are very small changes of the entropy under deformation, whereas the strain sensitivity of the internal energy is quite high, owing to the nature of the interatomic interactions (so that $\sigma \approx \rho \partial e / \partial \varepsilon$ );

(ii) other materials, such as elastomers (Mark and Lal 1982), can suffer very large reversible distortions of the macromolecular chains in the rubber elasticity regime, which strongly alters the entropy, almost without modification of the internal energy (so that $\sigma \approx-\rho T \partial s / \partial \varepsilon)$

For the first case, linear elasticity is generally an appropriate model; the elastic moduli are quite high and do not depend very much on temperature. On the contrary, rubber elasticity is characterized by much lower moduli (by a factor of $10^{3}-10^{4}$ on average) and a stronger temperature dependence. Unusual be- 


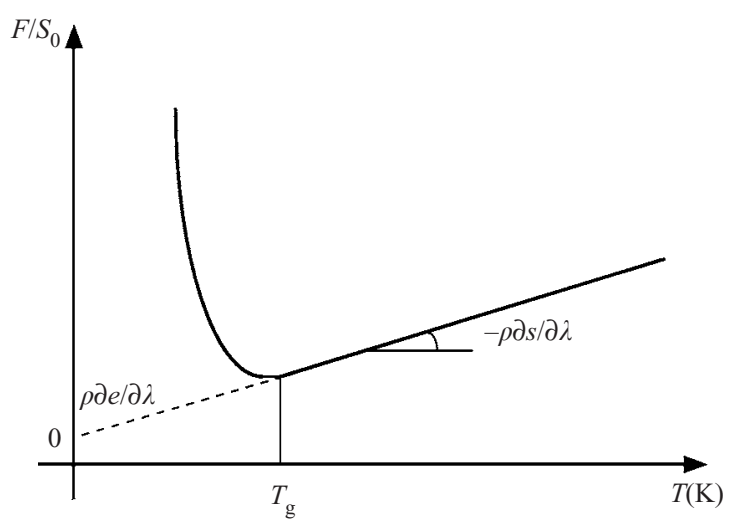

Figure 1

Temperature dependence of the force per unit area of the initial cross-section needed to maintain a given elongation for an elastomer sample.

havior, which can be understood easily from a uniaxial version of Eqn. (11) (i.e., $F \approx-\rho T \partial s / \partial \lambda$ ), is observed, such as shortening under load on heating or heat production by elongation. The entropic origin of rubber elasticity is easily proved by the temperature dependence of the force per unit area of the initial cross-section, $F / S_{0}$, needed to provoke a given elongation $\lambda$ (new length over initial length).

Typical experimental data obey a two-slope scheme (Fig. 1). Below the glass transition temperature, $T_{\mathrm{g}}$, the force abruptly decreases with increasing temperature, as observed for crystalline materials; rubber elasticity is dominant for $T \geqslant T_{g}$, as proved by the increasing (almost linear) variation of the stress with temperature (the slope, given by $-\rho \partial s / \partial \lambda$, is positive since the entropy decreases with an increasing elongation) and by the very low value of the back-extrapolated stress at $0 \mathrm{~K}$ from the rubber-type response $(-\rho \partial e / \partial \lambda \approx 0)$, which shows that the strain dependence of the internal energy is negligible.

\section{Heterogeneous Materials (Linear Thermoelasticity)}

This section is concerned with the prediction of the effective thermoelastic properties of heterogeneous materials, such as polycrystals or composite materials, from those of the constituents (grains or phases). Since the exact internal geometry of any representative volume element, $\Omega$, of such materials is generally not known, these properties can only be estimated or bounded. For the sake of simplicity, restriction is made to linear behavior, isothermal conditions (with $\tau=T-T_{0}$, a uniform change of temperature from the reference temperature, $T_{0}$, in the initial natural state), and homogeneous strain conditions, $\mathbf{E}$. The derivation of bounds for the overall elastic moduli and thermal expansion coefficients especially benefits from a thermodynamic treatment of elasticity.

(i) Since potentials are additive quantities, the overall total free energy, $F$, directly derives from the local one by integration

$$
\bar{\rho} F(\mathbf{E}, \tau)=\langle\rho f(\varepsilon, \tau)\rangle=\frac{1}{\Omega} \int_{\Omega} \rho f(\varepsilon, \tau) \mathrm{d} \Omega
$$

where $\bar{\rho}$ is the overall mass per unit volume and $\langle\ldots\rangle$ denotes a spatial average. Both at the local and the global levels, the free energy is quadratic and the constitutive equations are similar in form. From the local relationships

$$
\begin{gathered}
\boldsymbol{\Sigma}=\rho \frac{\partial f}{\partial \varepsilon}=\mathbf{C}: \varepsilon-\tau \phi, \quad s=-\rho \frac{\partial f}{\partial \tau}=\phi: \varepsilon+C_{v} \tau, \\
\phi=\mathbf{C}: \alpha
\end{gathered}
$$

where $C_{v}$ is the specific heat at constant strain, the following overall relationships can be derived (Stolz 1986):

$$
\begin{gathered}
\Sigma=\langle\sigma\rangle=\bar{\rho} \frac{\partial F}{\partial \mathbf{E}}=\mathbf{C}^{\mathrm{eff}}: \mathbf{E}-\tau \Phi^{\mathrm{eff}}, \\
\bar{\rho} s=\langle\rho s\rangle=-\bar{\rho} \frac{\partial F}{\partial \tau}=\Phi^{\mathrm{eff}}: \mathbf{E}+C_{v}^{\mathrm{eff}} \tau, \\
\Phi^{\mathrm{eff}}=\mathbf{C}^{\mathrm{eff}}: \alpha^{\mathrm{eff}}
\end{gathered}
$$

where the superscript "eff" refers to "effective" macroscopic quantities.

(ii) Owing to the linear character of the whole problem, the strain field, $\varepsilon(\vec{x})$, depends linearly on the loading parameters $(\mathbf{E}, \tau)$, i.e.,

$$
\varepsilon(\vec{x})=\mathbf{A}(\vec{x}): \mathbf{E}+\tau \eta(\vec{x})
$$

and a decomposition of the problem into two elementary ones, with $\mathbf{E}=0$ and $\tau=0$ respectively, can be carried out. This results in the following relationships:

$$
\begin{gathered}
\mathbf{C}^{\mathrm{eff}}=\langle\mathbf{C}: \mathbf{A}\rangle, \quad \Phi^{\mathrm{eff}}=\langle\phi: \mathbf{A}\rangle, \\
C_{v}^{\mathrm{eff}}=\left\langle C_{v}\right\rangle+\langle\phi: \eta\rangle
\end{gathered}
$$

These expressions show especially that the effective thermal expansion tensor, $\alpha^{\text {eff }}$, given by $\mathbf{C}^{\text {eff }}: \alpha^{\text {eff }}=$ $\langle(\mathbf{C}: \alpha): \mathbf{A}\rangle$, as well as the effective heat capacity at constant strain, $C_{v}^{\text {eff }}$, do not reduce to the spatial averages of the corresponding quantities. This results basically from the fact that the thermal strain field is generally not compatible, which is responsible for the presence of a thermal residual (self-equilibrated) stress 
field and its associated stored elastic energy. The foregoing treatment can be developed in order to derive bounds for $\alpha^{\text {eff }}$ and to perform a refined analysis of the thermal stresses, which cannot be obtained from a purely mechanical treatment of thermoelasticity.

\section{Conclusions}

The foregoing derivations have provided some illustrations of the benefits that can be gained from a thermodynamic treatment of elasticity. The main advantage lies in the fact that the temperature dependence of the elastic behavior is naturally taken into account so that the associated constitutive equations are valid for any thermodynamic conditions instead of being specified for particular ones (e.g., isothermal, adiabatic, etc.). Moreover, definite relationships can be derived between the elastic characteristics exhibited for different such conditions. This treatment yields a simple and meaningful interpretation of the entropic nature of rubber elasticity; in addition, it offers a comprehensive framework for the derivation of thermoelastic properties of heterogeneous materials, including the analysis of thermal stresses at the local level.

See also: Mechanics of Materials

\section{Bibliography}

François D, Pineau A, Zaoui A 1998 Mechanical Behaviour of Materials, Kluwer, Dordrecht, The Netherlands, Vol. 1

Germain P, Nguyen Q S, Suquet P 1983 Continuum thermodynamics. ASME J. Appl. Mech. 50, 1010-20

Mark J E, Lal J 1982 Elastomers and Rubber Elasticity. American Chemical Society, Washington, DC

Salençon J 1995 Mécanique du Continu. Ellipses, Paris, Vol. 2

Stolz C 1986 General relationships between micro and macroscales for nonlinear behaviour of heterogeneous media. In: Gittus J, Zarka J (eds.) Modelling Small Deformations of Polycrystals. Elsevier, London

A. Zaoui and C. Stolz 